\title{
La esgrima como ciencia matemática: el caso curioso de Luis Pacheco de Narváez
}

\section{Swordmanship as Mathematical Science: The Curious Case of Luis Pacheco de Narváez}

\section{Felix K. E. Schmelzer}

Universidad de los Andes

CHILE

fschmelzer@uandes.cl

[Hipogrifo, (issn: 2328-1308), 4.2, 2016, pp. 339-347]

Recibido: 07-03-2016 / Aceptado: 19-04-2016

DOI: http://dx.doi.org/10.13035/H.2016.04.02.22

Resumen. A partir del siglo XV, los representantes de las artes mecánicas adquieren una nueva seguridad en sí mismos y ya no desean que se les considere trabajadores manuales o artesanos. En este contexto, remiten con frecuencia al fondo matemático de su disciplina para elevarla del rango de arte mecánica al rango de arte liberal. En la España del Siglo de Oro, se concibe, entre otras, el arte militar como una disciplina matemática. Un caso particularmente curioso es el Libro de las grandezas de la espada (1600) cuyo autor, Luis Pacheco de Narváez (1570-1640), propaga la esgrima, a la que llama «verdadera destreza», como una ciencia basada en las artes del quadrivium. El presente artículo esboza brevemente la estrategia argumentativa con la que este autor quiere comprobar el estatus científico-matemático de su disciplina, basándose en un capítulo preliminar titulado «Los fundamentos sobre que está fundada la verdadera destreza».

Palabras clave. Pacheco, Siglo de Oro, esgrima, matematización de las artes, retórica.

Abstract. From the 15th century on, the practitioners of the mechanical arts obtain a new self-confidence and do no longer want to be regarded merely as manual workers. In this context, they frequently underline the mathematical basis of their discipline in order to increase its value from the status of a mechanical to that of

1. Una versión preliminar de este trabajo en Schmelzer, 2016, pp. 156-162. 
a liberal art. In the Spanish golden age period, the military art, amongst others, is conceived as a mathematical discipline. A particularly curious case is the Libro de las grandezas de la espada (1600) whose author, Luis Pacheco de Narváez (15701640), propagates the art of swordsmanship, which he names «the true skill», as a science based on the disciplines of the quadrivium. The present article outlines the arguments applied by Pacheco in a preliminary chapter in order to prove the mathematical and scientific status of his discipline.

Keywords. Pacheco, Spanish Golden Age, Swordsmanship, Mathematisation of the Arts, Rhetoric.

Leon Battista Alberti expone en el primer libro Della pittura los fundamentos matemáticos y, en particular, geométricos de este arte y afirma que solo quien conoce las características de las superficies puede ser un buen pintor ${ }^{2}$. Así, quiere elevar la pintura del rango de arte mecánica al rango de arte liberal. El genovés es pionero de un movimiento extendido en la temprana Edad Moderna: a partir del siglo XV, los representantes de las artes mecánicas adquieren una nueva seguridad en sí mismos y ya no desean que se les considere trabajadores manuales o artesanos ${ }^{3}$. En este contexto, remiten con frecuencia al fondo matemático de su disciplina, lo que se explica por el alto estimo del que gozan las disciplinas del quadrivium a lo largo de la revolución científica. En España, la importancia y alta valoración de las matemáticas se revela, en particular, a través de la fundación de la Academia Real Matemática por Felipe II en 15824.

En algunos casos, sin embargo, la supuesta relación del arte con las matemáticas parece bastante artificial, al menos desde la perspectiva de hoy. En la España del Siglo de Oro, se concibe, entre otras, el arte militar como una disciplina matemática, como muestra Diego de Álava (1557-1597) en su tratado El perfecto capitán, instruido en la disciplina militar y nueva ciencia de la artillería (1590), obra que se introduce a través de un panegírico exorbitante de la aritmética y la geometría ${ }^{5}$. La vinculación entre el arte militar y la geometría ya aparece en Platón ${ }^{6}$, pero en la obra de Álava es realzada de manera llamativa. Y hay casos más curiosos. Juan de Alcega, por ejemplo, titula un tratado de sastrería Libro de geometría práctica ${ }^{7}$ (1589) y concibe esta práctica artesanal como matemática aplicada. Otro caso particularmente interesante es el Libro de las grandezas de

\footnotetext{
2. «E voglio sia persuaso apresso di noi che solo colui sarà ottimo artefice, el quale arà imparato conoscere gli orli delle superficie e ogni sua qualità» (Della pittura, l, 23).

3. Esta nueva valoración de las artes mecánicas, y de los trabajos fundados en ellas, se hace muy evidente en La Piazza universale di tutte le professioni del mondo de Tommaso Garzoni (1589), una obra muy difundida a finales del siglo (traducida al castellano, con añadidos propios, bajo el título La plaza universal de todas ciencias y artes por Suárez de Figueroa en 1615).

4. Más informaciones sobre la fundación de la Academia se encuentran en Esteban Piñero y Vicente Maroto, 2002. En cuanto a la matematización de las artes en la España del siglo XVI, ver Flórez, 2001.

5. «De los admirables efectos de la arismética y geometría», fols. XIIV-XIVr.

6. República, $526 \mathrm{~d}$.

7. Esta obra, y también la de Álava, se encuentran en el corpus del Diccionario de la ciencia y de la técnica del Renacimiento (DICTER).
} 
la espada (1600) cuyo autor, Luis Pacheco8 (1570-1640), propaga la esgrima, que designa «verdadera destreza», como una ciencia basada en el quadrivium. En un capítulo preliminar titulado «Los fundamentos sobre que está fundada la verdadera destreza» ${ }^{9}$ intenta comprobar el estatus científico-matemático de su disciplina a través de una sofisticada estrategia argumentativa, que voy a analizar brevemente en lo que sigue ${ }^{10}$, para revelar que la matematización de las artes en el Siglo de Oro es también un fenómeno retórico.

Es obvio que la argumentación de Pacheco está marcada por los tratados matemáticos de su época, como se aprecia en expresiones como «fundamentos fuertes», «demostraciones tan evidentes e infalibles», «certeza y verdad», «cuenta y razón», etc ${ }^{11}$. Ya en el título del capítulo en cuestión se introduce la metáfora central del «fundamento» que el autor retoma en el primer párrafo, comunicando a sus lectores en tono de confianza: «es necesario que primero sepáis las partes desta verdadera destreza que queréis profesar, los fundamentos tan fuertes sobre que está fundada». Esta metáfora domina todo el texto, y de ella se deduce también el argumento principal en favor del estatus matemático de la esgrima: si el fundamento es fuerte, también lo que se construye sobre él lo es. Unas líneas después, el lector llega a saber cuál es precisamente este «fundamento fuerte» de la esgrima, nada menos que la verdad misma:

La primera parte y más principal que tan ilustre y valerosa la hace es ser cierta y verdadera, teniendo la propia verdad que las demás ciencias, por ser sus demonstraciones tan evidentes e infalibles. $Y$ es este nombre de verdad de tanta majestad y grandeza que en diciendo que una cosa lo es, ni hay más que decir, ni encarecimiento mayor que poner. Porque así como la verdad ella en sí es firme y cierta, así es cierto y firme lo que en ella se funda y promete, porque la certeza del efecto consiste en la certeza de la causa de do procede, pero cuando el principio o causa es falso e incierto, el efecto que dél procediere ha de ser falso e incierto.

La estrategia retórica es evidente: repitiendo palabras clave como «verdad», «firme» y «cierto», Pacheco da a entender que la esgrima es superior a las demás ciencias por sus «demonstraciones tan evidentes e infalibles». Las reminiscencias a las obras matemáticas contemporáneas, que asimismo declaran la superioridad de las artes del quadrivium debido a los criterios de certeza y demostración, se

8. Pacheco de Narváez, noble y militar, es conocido sobre todo a través de la obra mencionada, que fue bastante popular en su época. Escribió varios tratados de esgrima, de los cuales destaca también el Compendio de la filosofía y destreza de las armas de Jerónimo Carranza (1612) en el que sigue desarrollando sus teorías particulares, remitiéndose al «padre de la esgrima», Jerónimo de Carranza, que obtuvo en el siglo XVI el status de un héroe nacional. Junto a este, Pacheco es considerado el fundador de la esgrima española. En el ámbito literario es conocido sobre todo por su famosa enemistad con Quevedo, que en El Buscón (II,1) se burla claramente de la esgrima matemática (ver final del artículo).

9. Fols. Ir-IIIr.

10. Un análisis más detallado de la obra completa de Pacheco en cuanto a la matematización de la esgrima todavía está pendiente.

11. Como he mostrado en otra ocasión (Schmelzer, 2016), dichas expresiones son lugares comunes de la presentación de las artes del quadrivium en los prólogos de los tratados matemáticos del siglo XVI. 
muestran en este pasaje tanto en el nivel argumentativo como en el estilístico. Veamos, por ejemplo, como Marco Aurel, matemático alemán que introduce el álgebra en España, describe la aritmética en su Libro primero de aritmética algebrática (1552): «Que la ciencia matemática, entre todas las artes liberales [...], sea la más clara y la más cierta y necesaria, es cosa evidente, pues solo ella (y principalmente lo que en ella se trata) es la que con más claras y abundosas demonstraciones se demuestra» ${ }^{12}$. Estamos, pues, ante un notable ejemplo de una transferencia de términos y estructuras argumentativas provenientes de las matemáticas a un campo de saber ajeno a ellas.

A partir de ahí, Pacheco inicia un elogio largo y exorbitante de la verdad. El objetivo está claro: habiendo ya explicado a sus lectores que ella es el «fundamento» de la «verdadera destreza», puede transferir, en la segunda parte del párrafo, los atributos de la verdad a la esgrima:

De la verdad dice Marco Tulio que es tan grande su fuerza que ella por sí, sin ayuda de nadie, se defiende de las asechanzas de los que maliciosamente la quieren encubrir ${ }^{13}$. La destreza, por lo que tiene de verdad, hace el mesmo efecto, y siempre sale vitoriosa. La verdad es la que rige los cielos, alumbra la tierra, sustenta la justicia, gobierna la República, confirma lo que es cierto, y aclara lo que es dudoso: centro a do todas las cosas reposan, norte por do todo el mundo rige, blanco a do todos deben caminar. La destreza, por lo que alcanza y goza desta verdad cuanto le es posible, hace los mismos efectos porque ella rige a los hombres, enseñándoles como han de buscar su conservación y defensa por caminos ciertos, alumbra el entendimiento y lo satisface con sus demonstraciones evidentes, sustenta al hombre y le defiende la vida y honor cuando se la quiere quitar el malo, que este es uno de sus particulares efectos. Desta ciencia gobierna, avisando cómo y por donde se haya de procurar, confirma en certeza lo que hasta aquí estaba en opinión, aclara lo que no es cierto con su presencia, porque un contrario con otro se conoce mejor. Es centro a do todas las aficiones de los hombres, inclinados a las armas, pueden reposar, porque es el fin de lo que podrán desear y hallar. Es norte por el cual todos se han de regir si quieren acertar.

Aunque todavía no lo ha anunciado de manera explícita, queda cada vez más claro que el autor caracteriza a la esgrima como ciencia matemática. Similar a la tradición platónica, que concibe las matemáticas, por su exactitud, como un guía del alma filosófica hacia la verdad ${ }^{14}$, argumento estándar en favor de estas disciplinas durante el

12. Aurel, Marco, Libro primero de aritmética algebrática (1552), fol. IIr. La obra es la primera en tratar el álgebra en lengua española.

13. «O magnam uim ueritatis, quae contra hominem ingenia, callidatem, sollertiam contraque fictas omnium insidias facile se per se ipsa defendat!» (Cicerón, Pro Marco Caelio, 63b).

14. Comp. el séptimo libro de la República de Platón. Al inicio de este libro, se ilustra, a través de la famosa parábola de la cueva, que la contemplación de las ideas constituye el objetivo principal de la educación de los filósofos gobernantes. Dicha parábola da a entender que el alma filosófica puede y debe subir - paulatinamente y con precaución, para que la luz de la verdad no le ciegue- de la oscuridad y del engaño del mundo material (la cueva) a la luz de la verdad de las ideas inmateriales (el sol). El objetivo final de este ascenso persigue la contemplación de la idea del Bien, que, como se explica, es, a la vez, la causa de la verdad y de la belleza. A partir de ahí, el Sócrates platónico deduce las consecuencias peda- 
Renacimiento, da a entender en la cita que la esgrima «rige» los hombres «por caminos ciertos», que «alumbra el entendimiento y lo satisface con sus demonstraciones evidentes» y que es un «norte» para quién busca la certeza. Asimismo, la oposición certeza-opinión que se introduce aquí como argumento en favor de la esgrima, sirve a los autores contemporáneos como argumento cara a las matemáticas. En este sentido, Juan Cornejo, en su prólogo al Tratado de matemáticas de Juan Pérez de Moya (1573), describe las artes del quadrivium como «perfecto saber»-«perfecto» porque se trata de un saber demostrable - y «guía y luz de la filosofía natural, en la cual, con tratar de cosas llanas, palpables y caseras, han sido tantas y tan varias las opiniones cuanto a cada cual se le antojaba o agradaba su parecer» ${ }^{15}$.

Sigue el pasaje nuclear del texto, donde la esgrima («destreza») es proclamada explícitamente una ciencia matemática universal que reúne en sí aritmética, geometría (designada como «la matemática») y filosofía natural ${ }^{16}$ :

La otra parte, que no menos excelente la hace, es que está fundado este maravilloso edificio de la destreza sobre tales y tan fuertes fundamentos que ni el rigor del tiempo la consumirá ni jamás se envejecerá, ni perderá de su ser un punto. Porque de cualquiera parte que la miréis, la hallaréis que estriba sobre las sólidas murallas de las ciencias, las cuales le ayudan con la verdad que cada una por sí tiene y le favorecen con certeza y la hacen más fuerte, añadiendo fuerza a fuerza. De tal suerte que si la matemática en si tiene certeza y verdad, la destreza se muestra por ella, y la fortalece con sus demostraciones. Y si la arismética en si tiene verdad y certeza, la de la destreza se acompaña con ella porque trata de cuenta y razón. Y si la filosofía natural nos descubre y manifiesta muchos secretos, ella misma declara los que la destreza tiene.

Lo que Pacheco presenta aquí como argumento nuevo («la otra parte») no es sino una variación de lo anteriormente dicho. El autor insiste en la metáfora del «fundamento», que genera aquí toda una isotopía de «fuerza» y «certeza», con la única diferencia que ahora explica la «fuerza» de este fundamento precisamente por las matemáticas, obviamente sinónimo de verdad. La estructura argumentativa coincide en este caso con la literatura técnica de la época. Veamos, por ejemplo, como el famoso cartógrafo Pedro de Medina (1493-1567) introduce el arte de navegar a sus lectores:

entre las artes el arte de la navegación es más excelente que las otras, pues no solo comunica con ellas, mas incluye en sí las más principales, es, a saber: arit-

gógicas necesarias para alcanzar este objetivo y pregunta por las disciplinas adecuadas para conducir el alma hacia la verdad. Propone las artes matemáticas (aritmética, geometría, astronomía y armonía) porque, según él, participan de la verdad inmutable del ser eterno, por lo que estimulan la razón y la guían hacia la contemplación de las ideas. La aritmética, por ejemplo, lleva el alma a la verdad porque el ser del número hace intuir la unidad escondida detrás de la variedad de las apariencias del mundo material (525a-b). También, en la medida en que llevan de lo visible y audible a lo invisible e inaudible del mundo ideal, cumplen este papel pedagógico la geometría, la astronomía y la música.

15. «Prólogo del doctor Cornejo, médico, a los curiosos lectores», fol. IIIv.

16. La filosofía natural es concebida como una disciplina filial de la astronomía durante el siglo XVI y, por tanto, como una ciencia matemática. 
mética, geometría, astrología. Estas tienen excelencia entre las matemáticas por la demostración verísima que de sus conclusiones hacen ${ }^{17}$.

También en este caso, el valor de la propia disciplina se explica por el hecho de que contiene en sí las principales artes matemáticas. Si este argumento parece justificado en el caso de la náutica, que, de hecho, se basa en conocimientos de astronomía y cosmografía, parece dudoso, al menos, respecto al arte de la esgrima. Con el apartado citado, Pacheco acaba ya la 'comprobatio' del presunto estatus científico-matemático de su disciplina.

Sin duda, el lector se preguntará qué significa dicha curiosa concepción de la esgrima en concreto. Una mirada al Libro de las grandezas de la espada aclara que el militar, de hecho, intenta enseñar su disciplina como geometría aplicada. Después de explicar algunas de las cualidades que debe tener el caballero que practique esa 'ciencia'18, intenta adaptar los teoremas y postulados de los Elementos euclidianos al manejo de la espada, sobre todo en el segundo libro. Eso ya se revela en los títulos de los capítulos, por ejemplo: «Demostración matemática, en la cual se manifiesta como alcanza más el ángulo recto que otro alguno»; "Cuatro líneas que particularmente sirven en la destreza, así para las heridas como para los compases»; «Demostración del arco y la cuerda, en que se manifiesta la naturaleza del movimiento circular y recto»... Entre otros aspectos, trata la geometría del cuerpo humano con vistas a conocer los vínculos entre el brazo y el cuerpo necesarios para infligir heridas mortales. También, intenta desarrollar una teoría geométrica de los movimientos necesarios para el ataque y la defensa (en este contexto describe el «paso geométrico» ${ }^{19}$, por ejemplo), y explica varias proporciones de longitud entre las espadas (la propia y la del enemigo) ${ }^{20}$. El uso práctico de esas explicaciones es difícil de juzgar.

En fin, la obra de Luis Pacheco puede verse, desde la perspectiva de hoy, como una curiosidad científico-histórica y como una manifestación particularmente española del tópico de las armas y las letras. Esta obra, además, ha dejado huellas en la literatura de la época. Así, en el capítulo XVIII de la segunda parte de la famosa novela de Cervantes, don Quijote da una oración apasionada que se puede interpretar como parodia de los discursos seudo-científicos del Siglo de Oro. En ella, elogia la «ciencia de la caballería andante» como disciplina universal y subraya en este contexto que el que la profese «ha de saber las matemáticas, porque a cada paso se le ofrecerá tener necesidad dellas». Y las ideas particulares de Pacheco resuenan aún más en

17. Arte de navegar (1545), fol. IIIr. Es probablemente el tratado de navegación más difundido de su época. Fue editado en toda Europa en cinco idiomas durante los siglos XVI y XVII. Pacheco, sin duda, lo conocía.

18. Se mencionan tanto características físicas («la cabeza derecha», «ojos vivos y despiertos», «el pecho alto»...) como psíquicas (el caballero tiene que destacar en cuanto a su entendimiento, ingenio, memoria y prudencia).

19. «Paso geométrico ya dijimos, que ha de tener tres pies, uno de macizo, y los dos de hueco; y así está repartida en tres partes iguales, como lo señala de punto A a punto B y en las demás líneas» (fol. 66). 20. Es decir, dos espadas de igual longitud, espada corta contra espada larga, espada larga contra espada corta. 
el siguiente capítulo (XIX) de la segunda parte, donde don Quijote y Sancho se encuentran con «uno de estos a quien llaman diestros» que, después de haber derrotado a un adversario, les explica «las excelencias de la espada, con tantas razones demostrativas y con tantas figuras y demostraciones matemáticas, que todos quedaron enterados de la bondad de la ciencia». Asimismo, Quevedo, enemigo personal de Pacheco ${ }^{21}$, se burla directamente del maestro de armas en El Buscón. El primer capítulo de la segunda parte cuenta de un hombre aparentemente desorientado que, «mirando un libro, hacía unas rayas que medía con un compás», preguntando al protagonista «si iba a Madrid por línea recta, o si iba por camino circumflejo». Los dos pasan la noche en un albergue, donde el pobre hombre «empieza a andar a oscuras por el aposento, dando saltos y diciendo en lengua matemática mil disparates». El libro que lleva consigo se titula «Grandeza de la espada».

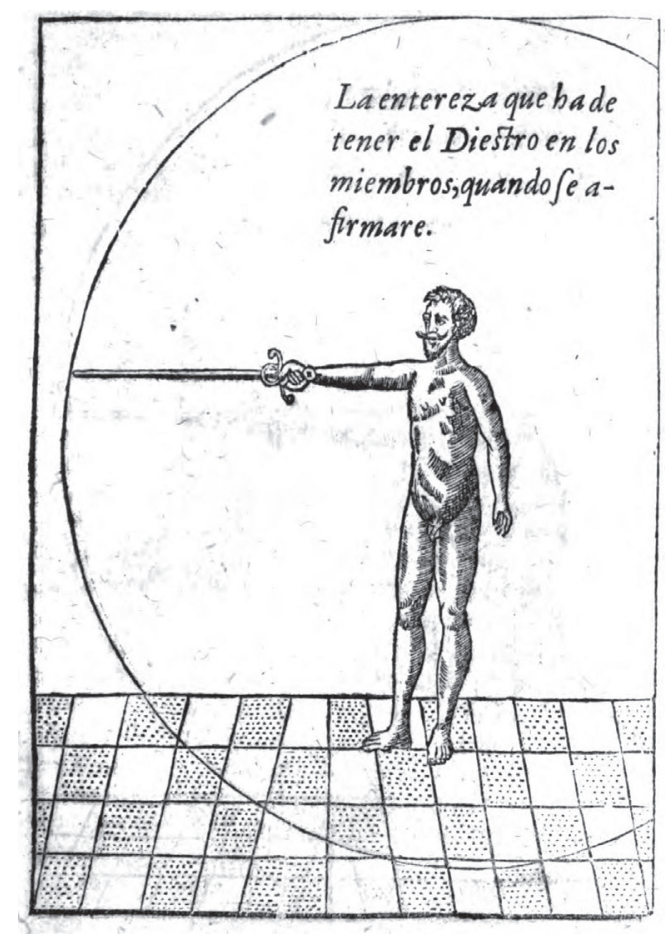

Libro de las grandezas de la espada, fol. 39r.

21. Me refiero a la anécdota famosa según la cual el poeta desenmascaró públicamente al maestro de armas por un diestro golpe de la espada del que este no podía defenderse. Pacheco, a su vez, «dirige en 1630 un memorial a la Inquisición en que denuncia Los Sueños, la Política de Dios, el Discurso de todos los diablos y el mismo Buscón» (Arellano, 2007, p. 4). 


\section{BIBLIOGRAFÍA}

Álava, Diego de, «De los admirables efectos de la arismética y geometría», en El perfecto capitán, instruida en la disciplina militar y nueva ciencia de la artillería, Madrid, Pedro Madrigal, 1590, fols. XIIv-XIVr.

Alberti, Leon Battista, Della pittura e della statua, Milano, Società Tipografica dei Classici Italiani, 1804.

Alcega, Juan de, Libro de geometría práctica, Madrid, Guillermo Drouy, 1589 (en DICTER).

Arellano, Ignacio, Introducción a «El Buscón», Alicante, Biblioteca Virtual Miguel de Cervantes, 2007.

Cervantes, Miguel de, Don Quijote de la Mancha, ed. Francisco Rico, Madrid, Prisa, 2014 [1615].

Cornejo, Juan, «Prólogo del doctor Cornejo, médico, a los curiosos lectores», en Pérez de Moya, Juan, Tratado de matemáticas, Álcala de Henares, Juan Gracián, 1573, fols. IIIv-Vr.

Diccionario de la ciencia y de la técnica del Renacimiento (DICTER), dir. María Jesús Mancho, Salamanca, Ediciones Universidad [dicter.usal.es].

Esteban Piñero, Mariano y María Isabel Vicente Maroto, «La Casa de Contratación y la Academia Real Matemática», en Historia de la ciencia y de la técnica en la Corona de Castilla, III. Siglos XVI y XVII, ed. José María López Piñero, Salamanca, Junta de Castilla y León, 2002, pp. 35-51.

Flórez, Cirilo, «Otra cara del humanismo», en Pórtico a la ciencia y a la técnica del Renacimiento, ed. María Jesús Mancho, Salamanca, Junta de Castilla y León, 2001, pp. 11-43.

Garzoni, Tommaso, La piazza universale di tutte le professioni del mondo; nuovamente ristampata; con l'aggionta d'alcune bellissime annotationi, Venetia, Guovanni Battista Somasco, 1589.

Medina, Pedro de, «Proemio del autor sobre el Arte de navegar, en que se declara la excelencia grande de la navegación», en Arte de navegar, Valladolid, Francisco Fernández de Córdoba, 1545, fols. IIIr-IVr (en DICTER).

Pacheco, Luis, «Los fundamentos sobre que está fundada la verdadera destreza», en Libro de las grandezas de la espada, Madrid, Juan Iñiguez de Lequerica, 1600 , fols. Ir-IIIr.

Pacheco, Luis, Libro de las grandezas de la espada, Madrid, Juan Iñiguez de Lequerica, 1600.

Pacheco, Luis, Compendio de la filosofía y destreza de las armas de Jerónimo Carranza, Madrid, Luis Sánchez, 1612.

Platón, República, trad. Conrado Eggers Lan, Madrid, Gredos, 2000. 
Quevedo, El Buscón, ed. Domingo Ynduráin, Madrid, Cátedra, 2014.

Schmelzer, Félix K. E., La retórica del saber. El prólogo de los tratados matemáticos en lengua española (1515-1600), New York, IDEA, 2016.

Suárez de Figueroa, Cristóbal, Plaza universal de todas ciencias y artes, Valladolid, Junta de Castilla y León, 2006 [1615]. 
